\title{
Prognosis of lithological variability of the water- blocking strata based on the results of complex interpretation of seismic and electrical data
}

\author{
Igor Sanfirov ${ }^{* 1}$, Yuri Stepanov ${ }^{1}$, Tatiana Baybakova ${ }^{1}$ and Alexander Taynitskiy ${ }^{1}$ \\ ${ }^{1}$ Mining Institute of the Ural Branch of the Russian Academy of Sciences, Branch of the Federal State \\ Budgetary Institution of Science of Perm Federal Research Center of the Ural Branch of the Russian \\ Academy of Sciences, Perm, Russia.
}

\begin{abstract}
This article deals with a comprehensive geophysical approach intended to ensure safety of mining operations at the Verkhnekamskoe field of potassium and magnesium salts. As an example, the article presents the results of additional detailed seismic and electrical studies performed on one of the mine fields of the Upper Kama potash-magnesium salt deposit (UKPMSD) within the site designed for mining operations. Based on the obtained results the structure in the form of a "dome" was identified. According to the data of the complex interpretation, the area of an indefinite lithology (most likely, associated with the replacement of salt deposits) was identified in the upstructure part.
\end{abstract}

\section{Introduction}

Seismic and electrical exploration methods take the lead within the complex of geophysical methods intended to ensure safety of mining operations at the Upper Kama potashmagnesium salt deposit (largest in Europe). The most popular seismic studies are studies that use the multifold reflection surveying procedure (MRSP) with the use of interference detection systems. As for the electrical exploration methods, the most popular are direct current methods in combination with the assessment of variability of natural polarization (NP): vertical electrical sounding (VES), symmetrical electrical profiling (SEP) and NP.

Seismic methods are mainly intended to be used for the determination of features of the physical and geological structure of the productive pool and its surrounding deposits. Electrical exploration methods allow to identify and assess negative hydrogeological processes in oversalt deposits. Such a combination of interpretative possibilities is relevant to existing and abandoned sites of a mine take of the salt mine. A complex interpretation of seismic and electrical exploration data allows to localize dangerous areas characterized by low elastic properties of the deposits in question and increased intensity of hydrogeological processes in the oversalt strata. Further such sites are considered as monitor objects

\footnotetext{
*Corresponding author: Sanf@mi-perm.ru
} 
controlled by using various types of monitoring and design of specialized mining engineering activities.

Combined interpretation conclusions for the sites with wave field problems within the salt strata are based on the laws of changes in propagation velocities of elastic waves and apparent electrical resistance in the similar intervals of the geologic section of oversalt deposits. For example, low values of propagation velocities of longitudinal elastic waves at high apparent resistances can often be caused by the presence of dry fractured intervals of the geologic section, whereas in case of low apparent resistances such values can be caused by similar but already watered areas. Such methodological approaches of a complex interpretation of geophysical data can be used for an advanced prognosis of geological structure features that influence safety of the designed mining operations.

\section{Materials and methods}

Seismic studies are carried out using the technique of non-explosive seismic exploration of a high resolution with the use of the interference detection system for the common depth point. The elastic vibrations were recorded by the seismic recorder "IS128", the number of active channels was 64 , the sampling interval was $0.2 \mathrm{~ms}$ and $0.5 \mathrm{~ms}$, the record length was 1500 samples, the magnification was $64 \mathrm{Db}$. The distance between the receiver points was 2 and $8 \mathrm{~m}$, the distance between the source points was 2 and $8 \mathrm{~m}$ as well. The 64-channel asymmetric variable system is used as the detection system.

Seismic materials were processed using SPS-PC and Express-OGT systems with the use of original programs of the active Seismoacoustics Department of the MI UB RAS. The materials were interpreted in the original complex developed by the Seismoacoustics Department of the MI UB RAS.

Electrical exploration studies in the lines of seismic profiles are carried out using the vertical electrical sounding (VES). The sounding was carried out using a symmetrical fourelectrode device fitted with the equipment of AMS-1 resistance method made with the help of employees of the Geophysics Department of Perm State University and LLC Scientific Production Enterprise "Intromag".

As an operating device, the symmetric four-electrode Schlumberger device was used. The distance between the transmitter electrodes and the center of the measuring device was sequentially increased with a progression of 1.25 . The maximum $\mathrm{AB}$ separation was 1000 $\mathrm{m}$, the $\mathrm{MN}$ detector line length was $5 \mathrm{~m}$ and $25 \mathrm{~m}$. The sounding interval was $100 \mathrm{~m}$.

The program IPI2Win developed by the Geophysics Department of Moscow State University was used for the interpretation of VES data.

\section{Results and discussions}

Additional detailed seismic and electrical exploration studies have been carried out on one of the mine fields of the UKPMSD within the site designed for mining operations.

According to the previous studies [1], a number of the most dynamically expressed seismic events is usually noticed on time sections (see Fig. 1, a) within the UKPMSD, which correspond to the target seismic reflecting horizons $(\mathrm{RH})$ associated with the lithophysical boundaries. The selected reflecting horizons are associated with the section of the lower part of the salt-marly strata, where the presence of the first continuous formations of rock salt (CFoRS*/salt "mirror") is possible; the location of the formation top E (Et) and sylvinite zone (Sil.), as well as with the marking clay (MC). 

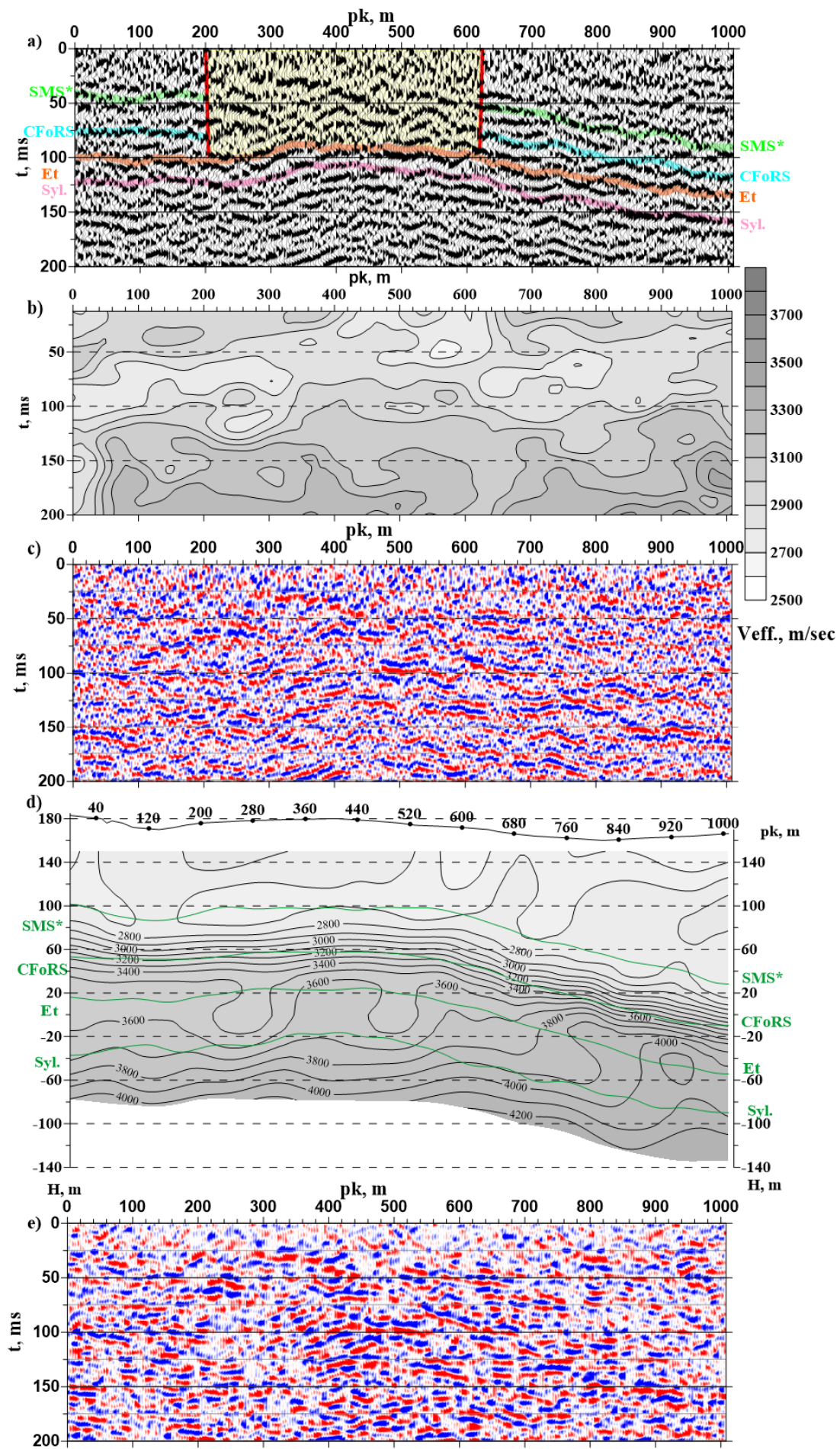

Fig. 1. Results of digital processing of the seismic data: a) CDP time section, b) velocity characteristic $(\mathrm{m} / \mathrm{s}), \mathrm{c})$ dynamic time section, d) seismo-geological section, e) migration 
Due to the presence of a negative vertical velocity gradient showing in the speed characteristics, RH SMS* was additionally introduced above RH CFoRS* in the section of the salt-marly strata (see Fig. 1, b). Such velocity boundary can be caused by vertical lithological differentiation in the salt-marly strata.

According to the results of seismic studies, a wave field problem in the form of a "dome" is identified in the section between the observation surface and the top of the sylvinite zone $(200-620 \mathrm{~m})$. In this range there is an increased velocity alternating differentiation both vertical and lateral in the velocity section (see Fig. 1, d) (in comparison with other parts of the profile). The reduced values of the elastic wave propagation velocities in the section of the salt-marly strata and carnallite zone on separate pieces of the profile line and partially within the limits of this problem are separated by a high-velocity formation in the salt-marly strata $(\mathrm{CFoRS} *$ - Et). Such areas are observed in the beginning $(0-260 \mathrm{~m})$ and end parts $(550 \mathrm{~m}$ and $>)$ of the profile. According to the depths, this formation corresponds to the possible position of the rock salt formations in the lower part of the salt-marly strata. Within the areas with low-velocity sections one can also see the increased attenuation of the intensity of the total wave field which is mostly obvious on the dynamic time sections (see Fig. 1, d). There is an increased intensity of scattered [2] waves (see Fig.1, e) on the "dome" borders, which may indicate the presence of certain vertically oriented contacts of deposits that differ in elastic properties.

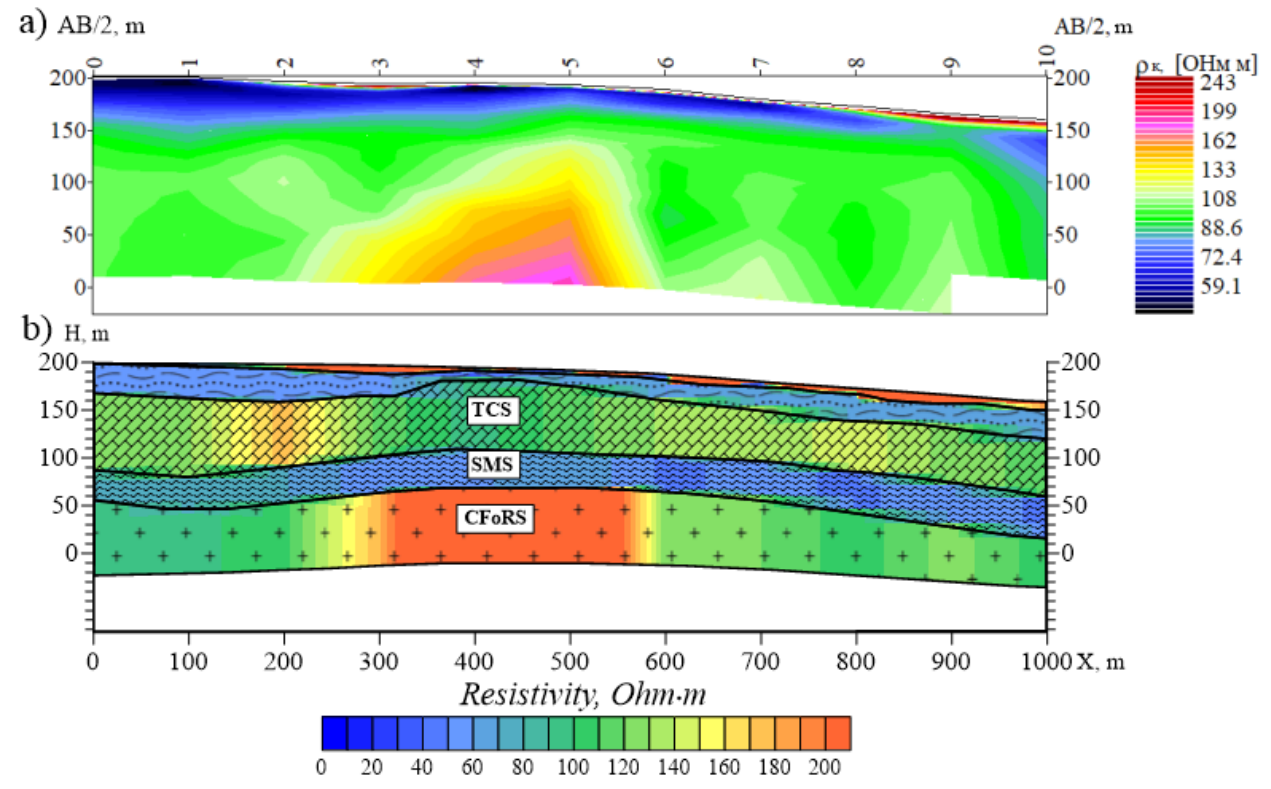

Fig. 2 Sections of apparent resistivity (AR) a) pk (Heff), b) geoelectric section

The section of apparent resistivity is based on the VES data, whereas the geoelectric section is based on the quantitative interpretation results (see Fig. 2).

On the section one can see a vertical variability of AR; there is an insignificant change of the section in the lateral direction. There are four formations in the oversalt deposits on the geoelectric section. Top of the fourth formation corresponds to the depths of occurring of the rock salt, which is the indicator horizon, immerses in the direction from west to east from the mark of $50 \mathrm{~m}$ to $-10 \mathrm{~m}$ respectively. According to the interpretation results, the specific electrical resistance (SER) is $110-150 \mathrm{Ohm} * \mathrm{~m}$. 


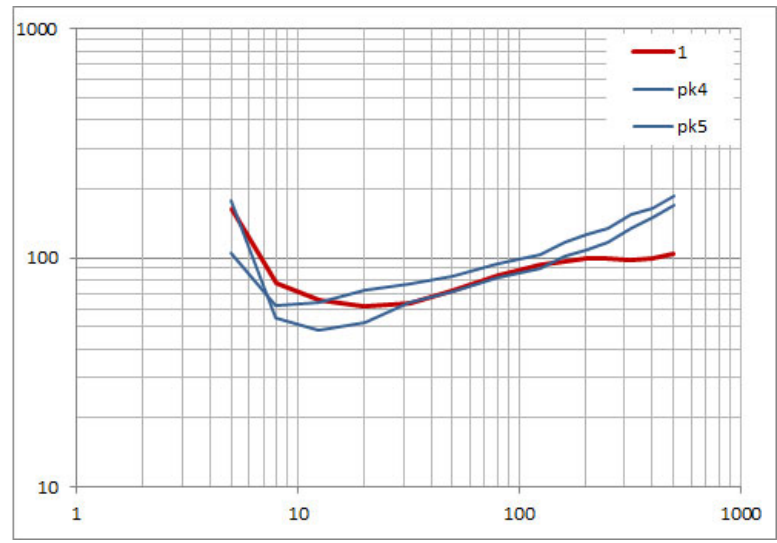

Fig. 3 Faired VES curve (1) and pk 4 and pk 5 VES curves

The analysis of VES curves shown in Fig. 3 indicates a significant difference between the faired VES curve and the apparent resistivity curves at the pickets 4 and 5 in the asymptotic part of the curve.

According to the results of electrical studies, the areas of increased resistances at the level of maximum separation were identified matching with the positive structural form selected in accordance with the seismic data. It should be noted that the features of the apparent resistance curves in the upstructure part can be interpreted as those caused by the lithological replacement in the depth range of the rock salt formations in the lower part of the salt-marly strata.

\section{Conclusions}

According to the results of complex interpretations of seismic and electrical data of the depth ranges of the upper formations of rock salt and in the upper part of the carnallite zone, (RH SMS*-CFoRS-Et) the area of an indefinite lithology was identified in the upstructure part. The replacement of the salt deposits with rocks with slightly lower elastic properties is assumed. At the same time the replacement contacts and salt deposits show a significant decrease of elastic properties (up to $10 \%$ ) and an increased dissipation of reflected waves.

The received results confirm the interpretative capabilities of the examined complex of geophysical methods and the appropriateness of a combined seismic and electrical exploration, including the areas designed for mining operations.

\section{References}

1. I.A. Sanfirov, A.I. Babkin, A.G. Yaroslavtsev, G.Yu. Priyma, K.B. Fatkin Seismic studies on conditions of potash deposit mining. / Geophysics. 5. P. 53-59. (2011).

2. I.A. Sanfirov, A.G. Yaroslavtsev, A.A. Zhikin, K.B. Fatkin, T.V. Baybakova Determination of shallow natural and technogenic heterogeneities of rock mass in different implementations of the interference field of reflected waves / Geophysics. 5. P. 56-63. (2017). 